A. CIMA, A. GASULL and F. MAÑOSAS (Barcelona)

\title{
LIMIT CYCLES FOR VECTOR FIELDS WITH HOMOGENEOUS COMPONENTS
}

Abstract. We study planar polynomial differential equations with homogeneous components. This kind of equations present a simple and well known dynamics when the degrees $(n$ and $m$ ) of both components coincide. Here we consider the case $n \neq m$ and we show that the dynamics is more complicated. In fact, we prove that such systems can exhibit periodic orbits only when $\mathrm{nm}$ is odd. Furthermore, for $\mathrm{nm}$ odd we give examples of such differential equations with at least $(n+m) / 2$ limit cycles.

1. Introduction. Consider the class of vector fields $X=(P, Q)$ in the plane with $P=P(x, y)$ and $Q=Q(x, y)$ homogeneous polynomials. From the dynamical point of view, there is a distinguished subclass: the subclass of $X=(P, Q)$ with $P$ and $Q$ homogeneous polynomials of the same degree. This is due to the fact that the behaviour of the orbits near the origin determines the global phase portrait. For this subclass, it is also known in which cases we have a focus or a centre at the origin:

Theorem $([1])$. The origin of $(\dot{x}, \dot{y})=(P(x, y), Q(x, y))$, where $P$ and $Q$ are homogeneous polynomials of the same degree, is of focus or center type if and only if $F(x, y)=x Q(x, y)-y P(x, y)$ is not zero for $(x, y) \neq(0,0) \in \mathbb{R}^{2}$. Furthermore, it is a center if and only if

$$
\left.\int_{0}^{2 \pi} \frac{x P(x, y)+y Q(x, y)}{F(x, y)}\right|_{x=\cos \theta, y=\sin \theta} d \theta=0 .
$$

In the general case the situation is much more complicated. We are interested in limit cycles and periodic orbits: In which cases can we get them? When are all the trajectories of the system periodic orbits? How

1991 Mathematics Subject Classification: 34C05, 58F21.

Key words and phrases: homogeneous function, vector field, limit cycle.

The authors are partially supported by a DGICYT grant number PB93-0860. 
many limit cycles can appear depending on the degrees of $P$ and $Q$ ? How to characterize the systems which have a center at the origin? Given the degrees of $P$ and $Q$, what is the cyclicity of a center or a weak focus inside this family?

It is clear that either the origin is the only critical point of $X$ or $X$ has a straight line of critical points. In this latter case we can reduce the study of the trajectories of $(\dot{x}, \dot{y})=X(x, y)$ to the study of the trajectories associated with another vector field $Y(x, y)$, also with homogeneous components, and with $\operatorname{deg} Y_{1}<\operatorname{deg} P$ and $\operatorname{deg} Y_{2}<\operatorname{deg} Q$. Hence for our purposes we will only consider the first case.

Our main result is the following:

Theorem A. Let $P$ and $Q$ be homogeneous polynomials with degree $n$ and $m$ respectively. Assume that $X=(P, Q)$ has the origin as an isolated critical point and that $n \neq m$. Consider the differential equation

$$
(\dot{x}, \dot{y})=(P(x, y), Q(x, y)) .
$$

Then the following hold:

(i) System (1) has no periodic orbits if either $n$ or $m$ are even.

(ii) If $n$ and $m$ are odd, then each of the following assertions is satisfied by some $P$ and $Q$ :

1. All solutions of (1) are periodic.

2. No solutions of (1) are periodic.

3. There are periodic and nonperiodic solutions of (1).

(iii) Denote by $N(n, m)$ the maximum number of limit cycles for a system of type (1) with $\operatorname{deg} P=n$ and $\operatorname{deg} Q=m$ both odd. Then $N(n, m) \geq$ $(n+m) / 2$.

Part (i) of the theorem easily follows from index theory. The proof of the second and third parts is based on the study of some families of vector fields of type (1) with centers and their perturbations inside the family. This part uses the usual technique of Abelian integrals in a suitable set of coordinates.

2. Proof of Theorem A. (i) Associated with a real isolated zero $p$ of a $\mathcal{C}^{\infty}$ map $X: \mathbb{R}^{n} \rightarrow \mathbb{R}^{n}$, there are two integer numbers, the index $\left(\operatorname{ind}_{p}(X)\right)$ and the multiplicity $\left(\mu_{p}(X)\right)$. It is proved in [3] that

$$
\operatorname{ind}_{p}(X) \equiv \mu_{p}(X)(\bmod 2) .
$$

If we take $p=0$ and $X=\left(P_{n}, Q_{m}\right)$, the fact that $X$ has polynomial components implies that the multiplicity coincides with the intersection number. Since 0 is an isolated critical point it is easy to prove that in our case $\mu_{0}(X)=n m$ (see [4]). Therefore

$$
\operatorname{ind}_{0}(X) \equiv n m(\bmod 2) .
$$


Remember that if a vector field has a unique critical point which is surrounded by a periodic orbit, then its index is 1 . Hence from the above congruence we see that periodic orbits can only appear for system (1) when $n m$ is an odd number.

(ii1) The Hamiltonian family of vector fields

$$
-y^{2 p-1} \frac{\partial}{\partial x}+x^{2 q-1} \frac{\partial}{\partial y}
$$

with Hamiltonian function $H(x, y)=p x^{2 q}+q y^{2 p}$ proves this case.

(ii2) It is not difficult to see that no trajectories of the vector field

$$
\left[-y^{2 p-1}+a x^{2 p-1}\right] \frac{\partial}{\partial x}+x^{2 q-1} \frac{\partial}{\partial y}, \quad a \neq 0, p>q,
$$

are periodic, using the Hamiltonian of the preceding paragraph as a Lyapunov function.

(ii3) Consider the family

$$
-y^{2 p-1} \frac{\partial}{\partial x}+\left[x^{3}+a x y^{2}\right] \frac{\partial}{\partial y}, \quad p \geq 3 .
$$

Remember that a characteristic at $0(\infty)$ is a curve that tends, with positive or negative time, to $0(\infty)$ with a definite slope, $\theta$. It is also known that when $X=(P, Q)$ is a polynomial vector field with $X(0,0)=(0,0)$ the only possible slopes which can have characteristics at zero (resp. at infinity) are the real roots of

$$
\begin{gathered}
x Q_{\min }(x, y)-\left.y P_{\min }(x, y)\right|_{(x=\cos \theta, y=\sin \theta)}=0 \\
\left(\operatorname{resp} . x Q_{\max }(x, y)-\left.y P_{\max }(x, y)\right|_{(x=\cos \theta, y=\sin \theta)}=0\right)
\end{gathered}
$$

where $\min =\min (\operatorname{deg}(P), \operatorname{deg}(Q)), \max =\max (\operatorname{deg}(P), \operatorname{deg}(Q))$ and $R_{k}$ denotes the homogeneous part of degree $k$ of a polynomial $R(x, y)$. It is not difficult to prove that if a real root of the above homogeneous functions has odd multiplicity then there always exists a characteristic associated with such a direction.

In our case the possible limit slopes for the characteristics are given by the zeros of $\left.x^{2}\left(x^{2}+a y^{2}\right)\right|_{(x=\cos \theta, y=\sin \theta)}$ at 0 , and of $\left.y^{2 p}\right|_{(x=\cos \theta, y=\sin \theta)}$ at $\infty$.

Therefore if $a<0$, then in a neighbourhood of 0 there are characteristics, and hence the system has orbits that are not closed curves. On the other hand, the fact that $x Q_{\max }-y P_{\max }=y^{2 p} \geq 0$ forces the orbits to turn around the origin in a neighbourhood of infinity. Note that it is clear that if $(x(t), y(t))$ is a trajectory solution of the differential equation associated with the system under consideration then $(x(-t),-y(-t))$ is also a trajectory. Therefore all periodic orbits near infinity are periodic. Hence it has been shown that the vector field considered has periodic and nonperiodic 
trajectories when $a<0$, and this finishes the proof. In fact, it can be proved that for $a \geq 0$ it has a global center.

(iii) First of all, we need to recall the generalized polar coordinates which were introduced by Lyapunov in his study of the stability of degenerate critical points (see [5]).

Let $x(\varphi)=\operatorname{Cs}(\varphi)$ and $y(\varphi)=\operatorname{Sn}(\varphi)$ be the solutions of the Cauchy problem $\dot{x}=-y^{2 p-1}, \dot{y}=x^{2 q-1}, x(0)=\sqrt[2 q]{1 / p}$ and $y(0)=0$. Then we have, among others, the following properties.

LEMMA 1. (i) $p \operatorname{Cs}^{2 q}(\varphi)+q \operatorname{Sn}^{2 p}(\varphi)=1$.

(ii) $d \operatorname{Sn}(\varphi) / d \varphi=\mathrm{Cs}^{2 q-1}(\varphi)$.

(iii) $d \operatorname{Cs}(\varphi) / d \varphi=-\operatorname{Sn}^{2 p-1}(\varphi)$.

(iv) $\operatorname{Cs}(-\varphi)=\operatorname{Cs}(\varphi)$.

(v) $\operatorname{Sn}(-\varphi)=-\operatorname{Sn}(\varphi)$.

(vi) $\operatorname{Cs}(\varphi)$ and $\operatorname{Sn}(\varphi)$ are $T_{p, q}$-periodic functions where

$$
T=T_{p, q}=2 p^{-1 / 2 q} q^{-1 / 2 p} \frac{\Gamma(1 /(2 p)) \Gamma(1 /(2 q))}{\Gamma(1 /(2 p)+1 /(2 q))},
$$

and $\Gamma$ denotes the gamma function.

(vii) $\operatorname{Cs}(T / 2-\varphi)=-\operatorname{Cs}(\varphi)$.

(viii) $\operatorname{Sn}(T / 2-\varphi)=\operatorname{Sn}(\varphi)$.

LEMma 2. We have $\int_{0}^{T} \operatorname{Sn}^{\alpha}(\varphi) \operatorname{Cs}^{\beta}(\varphi) d \varphi \neq 0$ if and only if $\alpha$ and $\beta$ are even.

Pr o of. Consider $\int_{0}^{T / 2} \operatorname{Sn}^{\alpha}(\varphi) \operatorname{Cs}^{\beta}(\varphi) d \varphi$ and set $\varphi=\omega-T / 2$. Then by using properties (iv)-(viii) we get

$$
\int_{0}^{T / 2} \operatorname{Sn}^{\alpha}(\varphi) \operatorname{Cs}^{\beta}(\varphi) d \varphi=(-1)^{\alpha+\beta} \int_{T / 2}^{T} \operatorname{Sn}^{\alpha}(\omega) \operatorname{Cs}^{\beta}(\omega) d \omega .
$$

From the above equality we have

$$
\int_{0}^{T} \operatorname{Sn}^{\alpha}(\varphi) \operatorname{Cs}^{\beta}(\varphi) d \varphi=\left[1+(-1)^{\alpha+\beta}\right] \int_{0}^{T / 2} \operatorname{Sn}^{\alpha}(\omega) \operatorname{Cs}^{\beta}(\omega) d \omega .
$$

So, if $\alpha+\beta$ is odd, then $\int_{0}^{T} \operatorname{Sn}^{\alpha}(\varphi) \operatorname{Cs}^{\beta}(\varphi) d \varphi=0$.

If $\alpha$ and $\beta$ are both odd then we can see that the statement is true by using the change $\varphi=T / 2-\omega$.

In order to prove our result we consider the change of variables $x=$ $\varrho^{p} \operatorname{Cs}(\varphi), y=\varrho^{q} \operatorname{Sn}(\varphi)$. Then

$$
H(x, y)=p x^{2 q}+q y^{2 p}=\varrho^{2 p q}\left(p \operatorname{Cs}^{2 q}(\varphi)+q \operatorname{Sn}^{2 p}(\varphi)\right)=\varrho^{2 p q} .
$$

The following result will finish the proof of Theorem A. 
THEOREM 3. For the vector field $-y^{2 p-1} \frac{\partial}{\partial x}+x^{2 q-1} \frac{\partial}{\partial y}$, we can find $a$ perturbation

$$
\left[-y^{2 p-1}+\varepsilon \bar{P}_{n}(x, y)\right] \frac{\partial}{\partial x}+\left[x^{2 q-1}+\varepsilon \bar{Q}_{m}(x, y)\right] \frac{\partial}{\partial y}
$$

with $\bar{P}_{n}$ (resp. $\left.\bar{Q}_{m}\right)$ homogeneous of degree $n=2 p-1$ (resp. $\left.m=2 q-1\right)$ which has $(n+m) / 2$ limit cycles.

P r o of. Using Pontryagin's method (see [6]), we know that each simple zero $R$ of

$$
F(\varrho)=\int_{H=\varrho^{2 p q}} \bar{P}_{n}\left(\varrho^{p} \operatorname{Cs}(\varphi), \varrho^{q} \operatorname{Sn}(\varphi)\right) d y-\bar{Q}_{m}\left(\varrho^{p} \operatorname{Cs}(\varphi), \varrho^{q} \operatorname{Sn}(\varphi)\right) d x
$$

produces a periodic orbit for the perturbed system $(\varepsilon \neq 0$, small enough) tending to $H(x, y)=R^{2 p q}$ as $\varepsilon \rightarrow 0$. Writing

$$
\bar{P}_{n}(x, y)=\sum_{i=0}^{n} a_{i} x^{i} y^{n-i} \quad \text { and } \quad \bar{Q}_{m}=\sum_{j=0}^{m} b_{j} x^{j} y^{m-j},
$$

we get

$$
\begin{aligned}
F(\varrho)= & \int_{0}^{T}\left\{\sum_{j=0}^{m} b_{j} \varrho^{p j+q(m-j)+p} \operatorname{Cs}^{j}(\varphi) \operatorname{Sn}^{2 p-1+m-j}(\varphi)\right. \\
& \left.+\sum_{i=0}^{n} a_{i} \varrho^{p i+q(n-i)+q} \operatorname{Cs}^{i+2 q-1}(\varphi) \operatorname{Sn}^{n-i}(\varphi)\right\} d \varphi \\
= & \varrho^{2 q^{2}+(p-q)}\left[\sum_{i=0}^{p+q-1} \alpha_{2 i} \varrho^{2 i(p-q)}\right]
\end{aligned}
$$

where we have used Lemma $2, \alpha_{k}=s_{k} \int_{0}^{T} \operatorname{Sn}^{2(p+q-1)-k}(\varphi) \operatorname{Cs}^{k}(\varphi) d \varphi$ and

$$
s_{k}= \begin{cases}b_{k}, & k=0,2, \ldots, 2 q-2, \\ a_{k-2 q+1}, & k=2 q, 2 q+2, \ldots, 2 q+2 p-2 .\end{cases}
$$

Taking $R=\varrho^{2(p-q)}$ we find that $F(\varrho)=0$ if and only if $\varrho=0$ or

$$
\bar{F}(R)=\alpha_{0}+\alpha_{2} R+\ldots+\alpha_{2(p+q-1)} R^{p+q-1}=0 .
$$

Then, by a suitable choice of $\alpha_{i}$ 's (that is, of $a_{i}$ 's and $b_{i}$ 's) we can get $p+q-1=(n+m) / 2$ simple zeros of $\bar{F}(R)$. From this the result follows.

3. Final remarks. For some subfamilies of the general case of $(P, Q)$ with homogeneous components we can give more information. The following two propositions are proved in [2]: 
Proposition 4. Consider the vector field

$$
\left[-y^{n}+\sum_{i=1}^{n} a_{i} x^{i} y^{n-i}\right] \frac{\partial}{\partial x}+x^{m} \frac{\partial}{\partial y}
$$

with $n=2 p-1>m=2 q-1$ and $p$ and $q$ positive integers. Then for the associated differential equation the following hold:

(a) The origin is a center if and only if $a_{1}=a_{3}=\ldots=a_{n}=0$.

(b) If $a_{1}=a_{3}=\ldots=a_{2 i-3}=0, a_{2 i-1} \neq 0$, then the origin is a weak focus and under perturbations inside the family it can produce $i-1$ limit cycles.

(c) The cyclicity of the center is $p-1=(n-1) / 2$.

Proposition 5. Consider the vector field

$$
-y^{n} \frac{\partial}{\partial x}+\left[x^{m}+\sum_{i=1}^{n} A_{i} x^{m-i} y^{i}\right] \frac{\partial}{\partial y}
$$

with $n=2 p-1>m=2 q-1$ and $p$ and $q$ positive integers. Then for the associated differential equation the following hold:

(a) A neighbourhood of infinity is filled with periodic orbits if and only if $A_{1}=A_{3}=\ldots=A_{m}=0$.

(b) If $A_{1}=A_{3}=\ldots=A_{2 i-3}=0, A_{2 i-1} \neq 0$, then the stability of the infinity is given by the sign of $a_{2 i-1}$. Furthermore, in this case, the maximum number of periodic orbits that bifurcate from infinity inside the family and for small perturbations is $i-1$.

(c) The maximum number of periodic orbits that can appear from infinity by perturbing the case $A_{1}=A_{3}=\ldots=A_{m}=0$ inside the family studied is $q-1=(m-1) / 2$.

In Theorem A(iii) we define the number $N(n, m)$ and prove that $N(3,1)$ $\geq 2$. One of the simplest problems that we have not been able to solve is to find if $N(3,1)=2$.

\section{References}

[1] J. Argémi, Sur les points singuliers multiples de systèmes dynamiques dans $\mathbb{R}^{2}$, Ann. Mat. Pura Appl. (4) 79 (1968), 35-69.

[2] A. Cima, A. Gasull and F. Mañosas, Cyclicity of a family of vector fields, J. Math. Anal. Appl. 196 (1995), 921-937.

[3] D. Eisenbud and H. Levine, An algebraic formula for the degree of a $C^{\infty}$ map germ, Ann. of Math. 106 (1977), 19-44.

[4] W. Fulton, Algebraic Curves. An Introduction to Algebraic Geometry, Benjamin, New York, 1969. 
[5] A. M. Lyapunov, Stability of Motion, Math. Sci. Engrng. 30, Academic Press, New York, 1966.

[6] L. S. Pontryagin, On dynamical systems close to the Hamiltonian ones, Zh. Eksperiment. Teoret. Fiz. 4 (1934), 883-885 (in Russian).

Departament de Matemàtica Aplicada II

E.T.S. d'Enginyers Industrials de Terrassa

Universitat Politècnica de Catalunya

Colom 11, 08222 Terrassa, Barcelona, Spain

E-mail: cima@ma2.upc.es
Departament de Matemàtiques, Edifici C Universitat Autònoma de Barcelona 08193 Bellaterra, Barcelona, Spain E-mail: gasull@mat.uab.es manyosas@mat.uab.es

Received on 7.12.1995;

revised version on 30.9.1996 